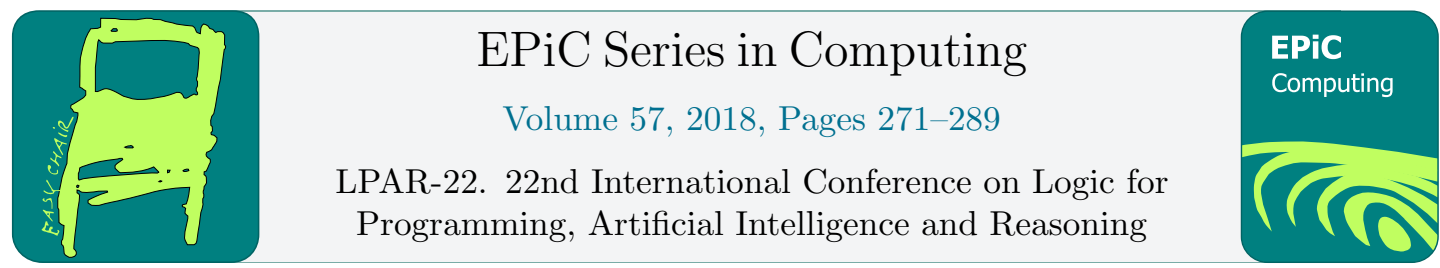

\title{
Left-Handed Completeness for Kleene algebra, via Cyclic Proofs *
}

\author{
Anupam Das ${ }^{1}$, Amina Doumane ${ }^{2}$, and Damien Pous ${ }^{2}$ \\ 1 University of Copenhagen \\ ${ }^{2}$ Univ Lyon, CNRS, ENS de Lyon, UCB Lyon 1, LIP
}

\begin{abstract}
We give a new proof that the axioms of left-handed Kleene algebra are complete with respect to language containments. This proof is significantly simpler than both the proof of Boffa (which relies on Krob's completeness result), and the more recent proof of Kozen and Silva. Our proof builds on a recent non-wellfounded sequent calculus which makes it possible to explicitly compute the invariants required for left-handed Kleene algebra.
\end{abstract}

\section{Introduction}

Kleene algebra is a finitely axiomatised quasi-equational theory over regular expressions [12], which admits formal languages and binary relations as free models: every equation which is universally valid in one of those models, or equivalently, whose two sides denote the same rational language, is provable from the axioms of Kleene algebra [7, 26, 19, 8]. This theorem is important in practice since it shows that the equational theory of Kleene algebra is decidable, and actually PSPACE-complete: it reduces to the problem of comparing rational languages. Thanks to the model of binary relations, Kleene algebra and its extensions have been used to reason abstractly about program correctness [21, 22, 3, 16, 2]. The aforementioned decidability result actually made it possible to automate reasoning steps in proof assistants [9, 25, 27].

Several axiomatisations have been studied in the literature, and the existing completeness proofs are all technically involved. Redko first proved that every purely equational axiomatisation must be infinite [28]. Conway then conjectured the completeness of fourteen 'classical' equations, plus a family of axioms indexed by finite semigroups [12, page 116]; Krob proved this result twenty years later, in a 137 pages long paper [26]. In the same year, Kozen proved completeness of a finite quasi-equational axiomatisation (which is called Kleene Algebra nowadays), using an ingenious proof [20] (12 pages) where automaton computations are replayed algebraically, using matrices. This axiomatisation contains two implications characterising the Kleene star:
(L) if $z+y x \leq x$ then $y^{*} z \leq x$;
(R) if $z+x y \leq x$ then $z y^{*} \leq x$.

*This work has been funded by the European Research Council (ERC) under the European Union's Horizon 2020 programme (CoVeCe, grant agreement No. 678157, and MiLC, grant agreement No. 753431).

G. Barthe, G. Sutcliffe and M. Veanes (eds.), LPAR-22 (EPiC Series in Computing, vol. 57), pp. 271-289 
By removing the second one one obtains so-called left-handed Kleene algebras, which are the structures we consider in the present paper, and whose completeness with respect to rational language inclusions was stated without proof by Conway [12, Chap. 12, Thm. 5]. Such structures are important because there are models where one of the implications of Kleene algebra is not satisfied [18, 24] (see also Ex. 1 below).

Boffa $[7,8]$ proved that completeness of left-handed Kleene algebra follows from completeness of Conway's axioms, thus requiring Krob's extensive proof [26, Cor. 15.15]. Kozen and Silva recently gave a shorter but still highly technical proof [23], by relying on the coalgebraic semantics of regular expressions: Brzozowski's derivatives [11]. We give a new proof in this work.

Removing the implication ( $\mathrm{R}$ ) above disqualifies Kozen's technique for proving completeness [20]: both (L) and (R) are needed to replay automaton algorithms algebraically. In fact, the key difficulty for left-handed completeness consists in finding invariants to exploit the remaining implication (L). A key feature of our proof is that we compute these invariants explicitly: we do not need to compute decompositions and solve systems of linear equations, as in [23].

Our completeness proof builds on a sequent-style calculus, HKA, which was proposed recently in [14]. This system admits 'cyclic' proofs, where proofs are finite graphs with cycles rather than trees or dags, and soundness and completeness for rational language inclusions holds under a simple correctness criterion. These cyclic proofs make it possible to represent computations on automata in a structured way, which we exploit here to compute invariants and to obtain completeness. This gives us a simple and direct proof, where the constructed derivations in left-handed Kleene algebra closely follow analogous computations on automata.

\section{Regular expressions and their proof systems}

\subsection{Regular expressions and Kleene Algebras}

We consider regular expressions over a finite alphabet $A$ :

$$
e, f::=e \cdot e|e+e| e^{*}|1| 0 \mid a \in A
$$

The set of regular expressions is denoted $\operatorname{Exp}_{A}$, and we often write $e f$ for $e \cdot f$. Each expression $e$ denotes a rational language $\mathcal{L}(e) \subseteq A^{*}$, defined in the usual way [17].

A left-handed Kleene algebra is a tuple $(K, 0,1,+, \cdot, *)$ where $(K, 0,1,+, \cdot)$ is an idempotent semiring (see App. A) and, writing $x \leq y$ as shorthand for $x+y=y$, we have:
(L) if $z+y x \leq x$ then $y^{*} z \leq x$;
(L') $1+x x^{*} \leq x^{*}$

Given two regular expressions $e, f$, we write $\ell \mathrm{KA} \vdash e \leq f$ if $e \leq f$ is derivable from the axioms of left-handed Kleene algebra (or, equivalently, is true in every left-handed Kleene algebra).

Together with the idempotent semiring axioms, axioms (L) and (L') amount to requiring that $y^{*} z$ is the least fixpoint of $x \mapsto z+y x$. These axioms admit several equivalent formulations (e.g. rules 'P1l', 'P2l' and 'P3l' in [12, page 103]). A few additional axioms are included in [23], but those are all derivable from the ones used here. The point of left-handed Kleene algebra is that the right-handed versions of axioms (L) and (L'), characterising $z y^{*}$ as the least fixpoint of $x \mapsto z+x y$, are omitted. This makes it possible to capture natural models, e.g. the following.

Example 1. Given a complete lattice $\langle X, \leq, \bigvee\rangle$, monotone functions $f: X \rightarrow X$ such that $f(x \vee y)=f(x) \vee f(y)$ and $f(\perp)=\perp$ form a left-handed Kleene algebra (where $\perp=\bigvee \varnothing$.). Addition is computed pointwise: $(f+g)(x)=f(x) \vee g(x)$; product is composition of functions: 
$(f \cdot g)(x)=f(g(x))$; Kleene star is obtained by iteration: $f^{*}(x)=\bigvee_{n \in \mathbb{N}} f^{n}(x)$ where $f^{0}(x)=x$ and $f^{n+1}(x)=f\left(f^{n}(x)\right)$. Such models do not, in general, satisfy the implication (R) from the introduction (see App. F). Also note that we must restrict to functions that distribute over finite suprema to obtain a semiring: otherwise we only have semi-distributivity on the right [18].

Left-handed completeness is the statement that language inclusion implies derivability in $\ell \mathrm{KA}$ :

Theorem 2 (Left-handed completeness [12, 8, 23]). If $\mathcal{L}(e) \subseteq \mathcal{L}(f)$ then $\ell \mathrm{KA} \vdash e \leq f$.

(The converse implication, soundness, holds by direct inspection of $\ell \mathrm{KA}$ axioms.) We reprove this theorem in the remainder of this paper. The difficulties occur when the expression on the left, $e$, contains starred sub-expressions. When such a sub-expression occurs immediately on the left at toplevel, we shall rely on the following variant of axiom $(\mathrm{L})$ :

Lemma 3 (Invariant lemma). For all regular expressions e, $\Gamma, X, I$,

$$
\text { if }\left\{\begin{array}{l}
\ell \mathrm{KA} \vdash \Gamma \leq I \\
\ell \mathrm{KA} \vdash e I \leq I \quad \text { then } \quad \ell \mathrm{KA} \vdash e^{*} \Gamma \leq X . \\
\ell \mathrm{KA} \vdash I \leq X
\end{array}\right.
$$

The reason for the choice of notation $\Gamma$ and $X$ becomes apparent in the next section when we deal with proof systems. The expression $I$ in the above lemma is an invariant for $e$ on the left (second assumption of the lemma). Deriving the inequality $e^{*} \Gamma \leq X$ requires us to 'guess' the right invariant $I$; in general, neither $\Gamma$ nor $X$ can be chosen for $I$, so some intermediate expression must be used. The main technical contribution of the present paper is in computing such invariants and showing that they fulfil the requirements of Lem. 3 above.

\subsection{The system HKA of cyclic proofs}

The sequent-style system HKA was introduced in [14]. It is a 'cut-free' proof system for inequalities between regular expressions and is well-suited for proof search; indeed it admits a PSPACE proof search procedure, which is optimal. HKA works with a form of hypersequents, recording slightly more structure than usual sequents, and proofs over this system may contain cycles. Both of these features are critical for cut-free completeness. We recall this system below.

Notation 4 (Lists and multisets). We denote lists (resp. finite multisets) by $x_{1}, \ldots, x_{n}$ (resp. $\left.x_{1} ; \ldots ; x_{n}\right\}$ ), where $\left\{x_{i}\right\}_{i \in[1, n]}$ are the elements. We also use comma (resp. semicolon) to denote list concatenation (resp. multiset union). We write $\varepsilon$ for the empty list. We let $\Gamma, \Delta$ range over lists of regular expressions, and $X, Y$ range over multisets of such lists. We implicitly interpret such lists and multisets as regular expressions by converting lists into products and multisets into sums. For instance, the multiset $a, b ; c$ is interpreted as the regular expression $a b+c$.

A hypersequent (or simply a sequent) is an expression $\Gamma \rightarrow X$. Its general form is thus:

$$
e_{1}, \ldots, e_{l} \rightarrow f_{11}, \ldots, f_{1 n_{1}} ; \cdots ; f_{m 1}, \ldots, f_{m n_{m}} .
$$

A hypersequent $\Gamma \rightarrow X$ is valid if $\mathcal{L}(\Gamma) \subseteq \mathcal{L}(X)$.

The rules of (leftmost) HKA are given in Fig. 1. In the modal rule $(a)$, we use the following notation: if $X=\Delta_{1} ; \ldots ; \Delta_{n}$, we write $e . X$ for the multiset $e, \Delta_{1} ; \ldots ; e, \Delta_{n}$. The rules presented in [14] are slightly more permissive: here we restrict to leftmost proofs, where logical rules only apply to regular expressions in head position of a list. We impose this condition syntactically since we do not need the more general setting of [14]. We also do not include the usual contraction rule, which is not required in the present work. 


\section{Left logical rules:}

$\overline{0, \Delta \rightarrow} 0-l \frac{\Delta \rightarrow X}{1, \Delta \rightarrow X} 1-l \quad \frac{e, f, \Delta \rightarrow X}{e \cdot f, \Delta \rightarrow X} \cdot-l \quad \frac{e, \Delta \rightarrow X \quad f, \Delta \rightarrow X}{e+f, \Delta \rightarrow X}+-l \frac{\Delta \rightarrow X \quad e, e^{*}, \Delta \rightarrow X}{e^{*}, \Delta \rightarrow X} *-l$

Right logical rules:

$$
\frac{\Gamma \rightarrow \Sigma ; X}{\Gamma \rightarrow 1, \Sigma ; X} 1-r \frac{\Gamma \rightarrow e, f, \Sigma ; X}{\Gamma \rightarrow e \cdot f, \Sigma ; X} \cdot-r \quad \frac{\Gamma \rightarrow e, \Sigma ; f, \Sigma ; X}{\Gamma \rightarrow e+f, \Sigma ; X}+-r \frac{\Gamma \rightarrow \Sigma ; e, e^{*}, \Sigma ; X}{\Gamma \rightarrow e^{*}, \Sigma ; X} *-r
$$

Identity, modal and structural rules:

$$
\overline{\rightarrow \varepsilon} \text { id } \quad \frac{\Gamma \rightarrow X}{a, \Gamma \rightarrow a . X}(a) \quad \frac{\Gamma \rightarrow X}{\Gamma \rightarrow \Delta ; X} w k
$$

Figure 1: The rules of leftmost HKA.

Definition 5 (HKA proofs). A preproof is a potentially infinite derivation built using the rules of Fig. 1. A proof is a preproof that is fair for $*-l$, i.e. where every infinite branch contains infinitely many $*-l$ steps. A proof is regular if it has finitely many distinct subtrees. We write HKA $\vdash^{\omega} \Gamma \rightarrow X$ if $\Gamma \rightarrow X$ admits a regular proof.

We will only work with regular proofs that are, furthermore, uniform: every occurrence of the same sequent concludes identical subproofs. (Every proof can be transformed into a uniform one.) We moreover exploit the following concrete representation of such proofs.

Lemma 6. Every regular and uniform proof can be represented as a finite tree with back-pointers to *-l steps: a finite derivation using the rules from Fig. 1 where leaves are either axioms (0-l, id) or pointers to one of their proper descendants corresponding to a $*-l$ step.

Proof. Along each infinite branch, fairness gives infinitely many $*-l$ steps, which occur on finitely many sequents by regularity; cut the branch using a back-pointer on the first such sequent visited twice. Now each branch is finite so that we have a finite tree (with back-pointers) by König's lemma. This proof unfolds to the same infinite tree as the starting proof, by uniformity.

Example 7. The following derivation represents a regular and uniform proof, where $\bullet$ is used for back-pointers. We use it as a running example in the rest of the paper. We denote it by $\Pi_{0}$.

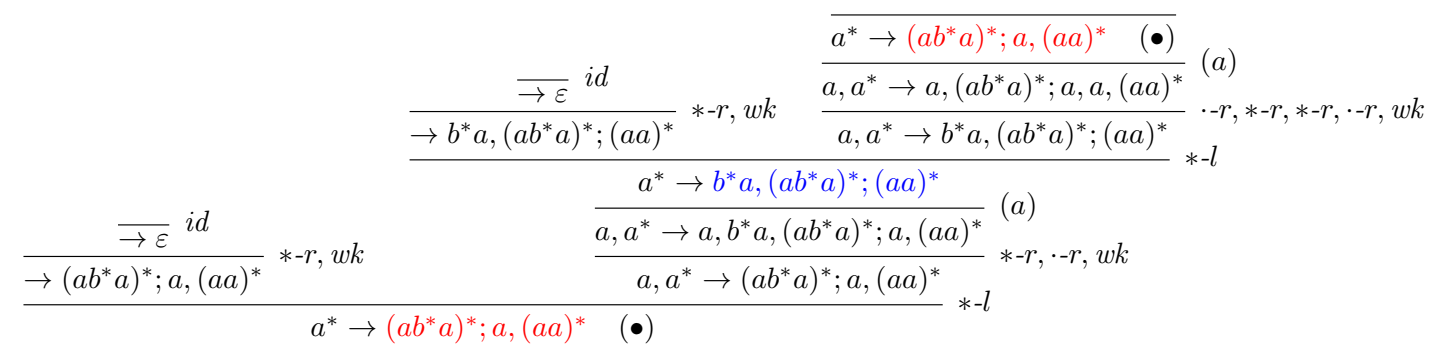

This proof intuitively corresponds to a computation where the language of the antecedent $\left(a^{*}\right)$ is explored and matched with the language of the succedent $\left(\left(a b^{*} a\right)^{*}+a(a a)^{*}\right)$, using an automaton construction reminiscent of partial derivatives [4]. Now introduce the following notations:

$$
\begin{array}{ll}
X_{0} \triangleq\left(a b^{*} a\right)^{*} ; a,(a a)^{*} & R_{0} \triangleq *-r, \cdot-r, w k \\
X_{1} \triangleq b^{*} a,\left(a b^{*} a\right)^{*} ;(a a)^{*} & R_{1} \triangleq{ }-r, *-r, *-r, \cdot-r, w k
\end{array} \quad R \triangleq *-r, w k
$$


We can write $\Pi_{0}$ and its subproof $\Pi_{1}$ rooted at $a^{*} \rightarrow X_{1}$ as follows:

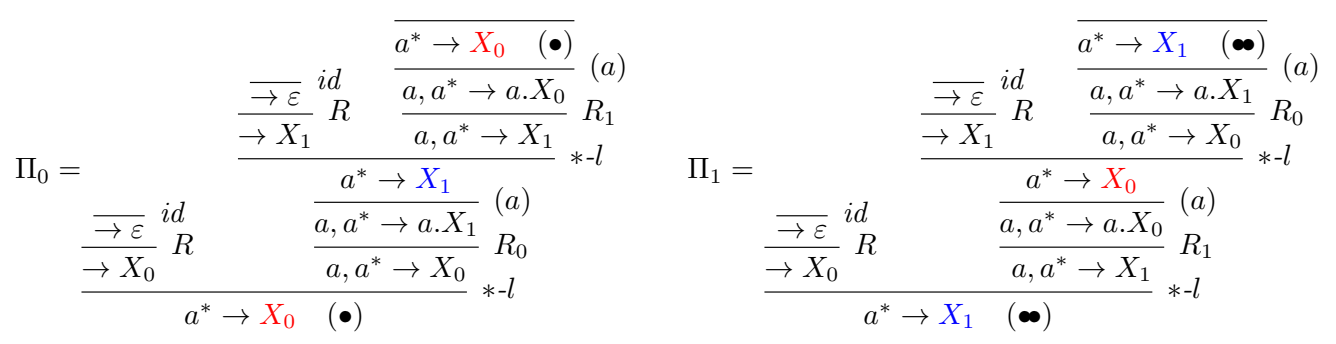

We give other examples in App. B. HKA is complete for rational language inclusions:

Theorem 8 (Completeness of HKA [14]). If $\mathcal{L}(\Gamma) \subseteq \mathcal{L}(X)$ then $\mathrm{HKA}^{\omega} \Gamma \rightarrow X$.

The proof of this theorem is not very difficult: logical rules of HKA are invertible so that we may apply them eagerly; when a normal form is reached, we use a weakening $(w k)$ followed by a modal step $(a)$, as usual in proof search for modal logics. We obtain regular proofs since the number of sequents appearing is bounded, by appealing to an order on the occurring expressions. The converse of Thm. 8 holds: HKA is sound [14]; this result also follows from Thm. 9 below.

\section{Proof of completeness}

To prove completeness of left-handed Kleene algebra, it suffices by Thm. 8 to translate (regular) proofs of HKA into $\ell \mathrm{KA}$ derivations:

Theorem 9 (Translation theorem). If $\mathrm{HKA}^{\omega} \vdash^{\omega} \Gamma \rightarrow X$ then $\ell \mathrm{KA} \vdash \Gamma \leq X$.

The rest of the paper is dedicated to the proof of this theorem. We first observe the following:

Proposition 10. If the premisses of a $\mathrm{HKA}$ rule are derivable in $\ell \mathrm{KA}$, so is its conclusion.

This means that every finite (well-founded) proof of HKA can be translated; the difficulty consists in handling cyclic - and thus infinite-proofs. To show Thm. 9, we need to slightly generalise it (Thm. 14 below), to deal with a certain form of hypotheses, which we define below.

Definition 11 (Hypotheses). A hypothesis is an expression $x \leq e$ where $x \in A$ and $e \in \operatorname{Exp}_{A}$. Let $\mathcal{H}$ be a set of hypotheses. We denote by $\ell \mathrm{KA}+\mathcal{H}$ the union of $\ell \mathrm{KA}$ axioms and $\mathcal{H}$. We write $\mathrm{HKA}+\mathcal{H}$ for the system obtained from HKA by adding, for every $(x \leq e) \in \mathcal{H}$, the following rule:

$$
\overline{x \rightarrow e} \mathcal{H}
$$

A straightforward induction yields the following lemma.

Lemma 12. Suppose $x \in \mathcal{L}(e)$ for a letter $x$. Then $\mathrm{HKA}^{\omega} \vdash^{-} x \rightarrow e$ and $\ell \mathrm{KA} \vdash x \leq e$.

Proviso 13. Let $\mathcal{H}_{0} \triangleq\{x \leq e \mid x \in \mathcal{L}(e)\}$. We assume that all sets of hypotheses contain $\mathcal{H}_{0}$ and that in HKA proofs, the modal rule $(a)$ is applied only when $\Gamma \neq \varnothing$ : instances of this rule breaking this assumption can be removed using hypotheses from $\mathcal{H}_{0}$.

Our goal is now to prove the following generalisation of Thm. 9:

Theorem 14. If HKA, $\mathcal{H} \vdash^{\omega} \Gamma \rightarrow X$, then $\ell \mathrm{KA}, \mathcal{H} \vdash \Gamma \leq X$. 
Thm. 8 follows by taking $\mathcal{H}=\mathcal{H}_{0}$; note that while Thm. 9 actually yields an equivalence, the converse of Thm. 14 does not hold - see App. D.

We prove Thm. 14 by induction on the lexicographical order $<_{l e x}$ on $\langle \# \Gamma, \# \pi\rangle$, where $\# \Gamma$ is the number of the $*$-subexpressions occurring in $\Gamma$, and $\# \pi$ is the number of (distinct) nodes in the starting regular proof $\pi$. We proceed by analysing the last rule $\rho$ applied in $\pi$.

If $\rho$ is the $\mathcal{H}$ rule, the result is immediate. Suppose that $\rho$ is a HKA rule which is not $*-l$, and let $\left\{\pi_{i}\right\}_{i}$ be the sub-proofs of $\pi$ rooted at the premisses of $\rho$. Let $\Gamma_{i} \rightarrow X_{i}$ be the conclusion sequent of $\pi_{i}$. Observe that $\# \Gamma_{i} \leq \# \Gamma$. Since $\rho$ is not a $*-l$, there is no back-pointer from $\pi_{i}$ to the conclusion of $\pi$, thus $\# \pi_{i}<\# \pi$. We have $\left\langle \# \Gamma_{i}, \# \pi_{i}\right\rangle<_{l e x}\langle \# \Gamma, \# \pi\rangle$, and we can apply the induction hypothesis to $\pi_{i}$. We conclude using Prop. 10.

Suppose now that $\rho$ is $*-l$, so that $\pi$ is of the following form:

$$
\frac{\frac{\pi_{1}}{\Gamma \rightarrow X} \quad \frac{\pi_{2}}{e, e^{*}, \Gamma \rightarrow X}}{e^{*}, \Gamma \rightarrow X} *-l
$$

We cannot in general apply the induction hypothesis to $\pi_{2}$ : we have $\#\left(e, e^{*}, \Gamma\right) \geq \#\left(e^{*}, \Gamma\right)$ and, since $\pi_{2}$ might have back-pointers to the conclusion of $\pi$, we might have $\# \pi_{2}=\# \pi$.

Instead, to find a derivation of $e^{*}, \Gamma \leq X$ in $\ell \mathrm{KA}+\mathcal{H}$, we use the invariant lemma (Lem. 3). A naive idea is to use $X$ as invariant, which would require us to show that $\ell \mathrm{KA}, \mathcal{H} \vdash \Gamma \leq X$ and $\ell \mathrm{KA}, \mathcal{H} \vdash e X \leq X$. The first inequality can be obtained by applying the induction hypothesis to $\pi_{1}$ (since $\# \Gamma<\#\left(e^{*}, \Gamma\right)$ ). Let us show the second inequality in the simple case where $\pi$ has the following property:

$$
\text { whenever a sequent } e^{*}, \Gamma \rightarrow Y \text { appears in } \pi \text {, we have } Y=X \text {. }
$$

In this case, if we replace $e^{*}, \Gamma$ by $X$ in all the antecedents of sequents in $\pi_{2}$, we can close all the back-pointers to $e^{*}, \Gamma \rightarrow X$ by an identity rule as shown below.

$$
\frac{\frac{\vdots}{e^{*}, \Gamma \rightarrow X}}{\frac{\vdots \pi_{2}}{e, e^{*}, \Gamma \rightarrow X}} \leadsto \frac{\frac{X \rightarrow X}{X} i d}{\frac{\vdots \pi_{2}\left[\left(e^{*}, \Gamma\right) / X\right]}{e, X \rightarrow X}}
$$

We conclude by applying the induction hypothesis to $\pi_{2}\left[\left(e^{*}, \Gamma\right) / X\right]$, since $\#(e, X)<\#\left(e^{*}, \Gamma\right)$.

Unfortunately, $\pi$ may contain sequents of the form $e^{*}, \Gamma \rightarrow X^{\prime}$ where $X^{\prime} \neq X$ (e.g. as in Ex. 7). In such a case, when we replace $e^{*}, \Gamma$ by $X$ in $\pi_{2}$, we get stuck with sequents of the form $X \rightarrow X^{\prime}$, for which we potentially do not even have $\mathcal{L}(X) \subseteq \mathcal{L}\left(X^{\prime}\right)$ :

$$
\frac{\frac{\vdots}{e^{*}, \Gamma \rightarrow X^{\prime}} \frac{\vdots}{e^{*}, \Gamma \rightarrow X}}{\frac{\vdots \pi_{2}}{e, e^{*}, \Gamma \rightarrow X}} \leadsto \frac{\frac{?}{X \rightarrow X^{\prime}} \frac{\vdots^{2}\left[\left(e^{*}, \Gamma\right) / X\right]}{X, X \rightarrow X}}{\frac{e, X}{d i}}
$$

If we had an intersection operator in our syntax, we could choose $X \cap X^{\prime}$ as invariant. Since we do not have such an operator, the challenge is to find an expression which will play the 
role of the intersection and for which we can derive the three inequalities required for an invariant, cf. Lem. 3. Note that blindly computing a regular expression for the rational language $\mathcal{L}(X) \cap \mathcal{L}\left(X^{\prime}\right)$ does not work since we would not know how to derive those three inequalities.

We handle this case in the next two sections: let us fix a regular proof $\underline{\pi}$ of $\underline{e}^{*}, \underline{\Gamma} \rightarrow \underline{X}_{1}$ that ends with a $*$-l step. Let $\underline{X}_{1}, \ldots, \underline{X}_{n}$ be an enumeration of the multisets such that $\underline{e}^{*}, \underline{\Gamma} \rightarrow \underline{X}_{i}$ is a conclusion of a $*-l$ step in $\pi$. In the following sections, we compute an expression $\underline{\mathcal{I}}$, the invariant, which intuitively corresponds to the intersection of $\underline{e}^{*} \underline{\Gamma}$ and $\underline{X}_{1}, \ldots, \underline{X}_{n}$ (Sect. 4), and we derive the following inequalities (resp. in Sect. 5.1, 5.2 and 5.3), as required by Lem. 3:

$$
\ell \mathrm{KA}, \mathcal{H} \vdash \underline{\Gamma} \rightarrow \underline{\mathcal{I}} \quad \ell \mathrm{KA}, \mathcal{H} \vdash \underline{e}, \underline{\mathcal{I}} \rightarrow \underline{\mathcal{I}} \quad \ell \mathrm{KA}, \mathcal{H} \vdash \underline{\mathcal{I}} \rightarrow \underline{X}_{1}
$$

We have used underlined variables $\underline{\pi}, \underline{e}, \underline{\Gamma}, \underline{X}_{i}, \underline{\mathcal{I}}$, to distinguish these global variables of the external induction from the local variables we will use in the subsequent lemmas and propositions.

\section{Computing the invariant}

For every $i \in[1, n]$, let $\underline{\pi}_{i}$ be the subproof of $\underline{\pi}$ rooted at $\underline{e}^{*}, \underline{\Gamma} \rightarrow \underline{X}_{i}$. To construct the invariant, we proceed in three steps:

1. First, we 'synchronise' the proofs $\left\{\underline{\pi}_{i}\right\}_{i}$ by taking their product. This product is a structure that we call a synchronised proof.

2. Second, we convert this synchronised proof into a non-deterministic automaton (NFA).

3. Third, we extract the invariant $\mathcal{I}$ from this NFA.

Several standard algorithms make it possible to extract regular expressions from finite automata (e.g. state removal from Kleene's theorem). However, here we need to design our own algorithm to retain control of the produced expressions, to derive the three inequalities of an invariant.

\subsection{Synchronised proofs}

We let $\mathcal{X}, \mathcal{Y}$ range over $n$-tuples of multisets (of lists of regular expressions).

Definition 15 (Synchronised proof system). A synchronised sequent is an expression of the form $\Gamma \Rightarrow \mathcal{X}$. A synchronised proof is a (potentially infinite yet regular) derivation built using the rules of Fig. 2, which is fair for $*-l$, as in Def. 5. As for HKA, we implicitly work with finite representations of synchronous proofs as trees with back-pointers, under Lem. 6 .

Intuitively, a synchronised proof of $\Gamma \Rightarrow X_{1}, \ldots, X_{n}$ is just a product of proofs for the sequents $\Gamma \rightarrow X_{i}$. Left logical rules are applied synchronously: at most one left logical rule applies for a given antecedent $\Gamma$; right logical rules are applied independently.

If $\Gamma \Rightarrow X_{1}, \ldots, X_{n}$ is a synchronised sequent, we call the sequent $\Gamma \rightarrow X_{i}$ its $i^{t h}$ projection. When we replace the synchronised sequents of a synchronised proof $\pi$ by their $i^{t h}$ projection and remove irrelevant right steps, we get an HKA $+\mathcal{H}$ proof, which we call the $i^{\text {th }}$ projection of $\pi$.

Conversely, one can construct synchronous proofs from independent ones:

Definition 16. Let $\left\{\pi_{i}\right\}_{i \in[1, n]}$ be a collection of HKA $+\mathcal{H}$ regular proofs, where $\Gamma \rightarrow Y_{i}$ is the conclusion of $\pi_{i}$ and $\rho_{i}$ is the last rule applied in $\pi_{i}$; (note that all $\pi_{i}$ have conclusion with the same antecedent $\Gamma$ ). Let $\prod_{i \in[1, n]} \pi_{i}$ be the synchronous proof defined coinductively as follows: 


\section{Left rules:}

$$
\frac{\left\{\Gamma_{i} \Rightarrow \mathcal{X}\right\}_{i \in I}}{\Gamma \Rightarrow \mathcal{X}} l \quad \text { If the following is a left HKA rule: } \quad \frac{\left\{\Gamma_{i} \rightarrow X\right\}_{i \in I}}{\Gamma \rightarrow X} l
$$

Right rules: (including weakening)

$$
\frac{\Gamma \Rightarrow \mathcal{X}, X, \mathcal{Y}}{\Gamma \Rightarrow \mathcal{X}, Y, \mathcal{Y}} r \quad \text { If the following is a right HKA rule: } \quad \frac{\Gamma \rightarrow X}{\Gamma \rightarrow Y} r
$$

Identity, hypothesis, and modal rules:

$$
\overline{\Rightarrow \varepsilon, \ldots, \varepsilon} \text { id } \quad \quad \quad \frac{\Gamma \Rightarrow X_{1}, \ldots, X_{n}}{x \Rightarrow e_{1}, \ldots, e_{n}} \mathcal{H}(a)
$$

Figure 2: The rules of synchronised HKA; the rule $\mathcal{H}$ applies only when the hypothesis $x \leq e_{i}$ belongs to $\mathcal{H}$ for every $i \in[1, n]$.

- If some $\rho_{i}$ is a right rule, let $k \in[1, n]$ be least such that $\rho_{k}$ is a right rule, say $r$. Supposing $r$ has premiss $\Gamma \rightarrow Z_{k}$ and $\theta_{k}$ is the sub-proof of $\pi_{k}$ rooted at $\Gamma \rightarrow Z_{k}$, we set:

$$
\prod_{i \in[1, n]} \pi_{i} \triangleq \frac{\prod_{i \in[1, k-1]} \pi_{i} \times \theta_{k} \times \prod_{i \in[k+1, n]} \pi_{i}}{\Gamma \Rightarrow Y_{1}, \ldots, Y_{k-1}, Z_{k}, Y_{k+1}, \ldots, Y_{n}} r
$$

- If all the rules $\rho_{i}$ are left logical rules, they are necessarily the same HKA rule $l$. Let $\left\{\Gamma_{j}\right\}_{j \in J}$ be the antecedents of the premisses of $l$, and let $\theta_{i j}$ be the sub-proof of $\pi_{i}$ rooted at $\Gamma_{j} \rightarrow Y_{i}$. We set:

$$
\prod_{i \in[1, n]} \pi_{i} \triangleq \frac{\left\{\frac{\prod_{i \in[1, n]} \theta_{i j}}{\Gamma_{j} \Rightarrow Y_{1}, \ldots, Y_{n}}\right\}_{j \in J}}{\Gamma \Rightarrow Y_{1}, \ldots, Y_{n}} l
$$

- If all the rules $\rho_{i}$ are the modal rule $(a)$, let $\Delta \rightarrow Z_{i}$ be the premiss of $\rho_{i}$ in $\pi_{i}$ and $\theta_{i}$ be the sub-proof of $\pi_{i}$ rooted in $\Delta \rightarrow Z_{i}$. We set:

$$
\prod_{i \in[1, n]} \pi_{i} \triangleq \frac{\prod_{i \in[1, n]} \theta_{i}}{\frac{\Delta \Rightarrow Z_{1}, \ldots, Z_{n}}{\Gamma \Rightarrow Y_{1}, \ldots, Y_{n}}}(a)
$$

- If all the rules $\rho_{i}$ are the hypothesis rule $\mathcal{H}$ (resp. the identity rule), we use the synchronised hypothesis rule (resp. identity rule).

Thanks to Proviso 13, if $\Gamma$ is of the form $\Gamma=a, \Delta$, the rules $\rho_{i}$ are either all hypothesis rules (if $\Delta=\varnothing$ ) or all modal rules (otherwise). Our definition thus covers all the possible cases.

Remark 17. Note that the $i^{\text {th }}$ projection of $\prod_{i \in I} \pi_{i}$ is an unfolding of $\pi_{i}$. The above product of proofs can be seen as a way to find a 'common unfolding' for them, as illustrated by Ex. 18. Also we point out that, in the first case of the definition above, when some $\rho_{i}$ are right rules, the precise choice of sequentialisation of right rules is not important for the sequel.

Example 18. The product of the proofs $\Pi_{0}$ and $\Pi_{1}$ from Ex. 7 is given on the left of Fig. 3. 


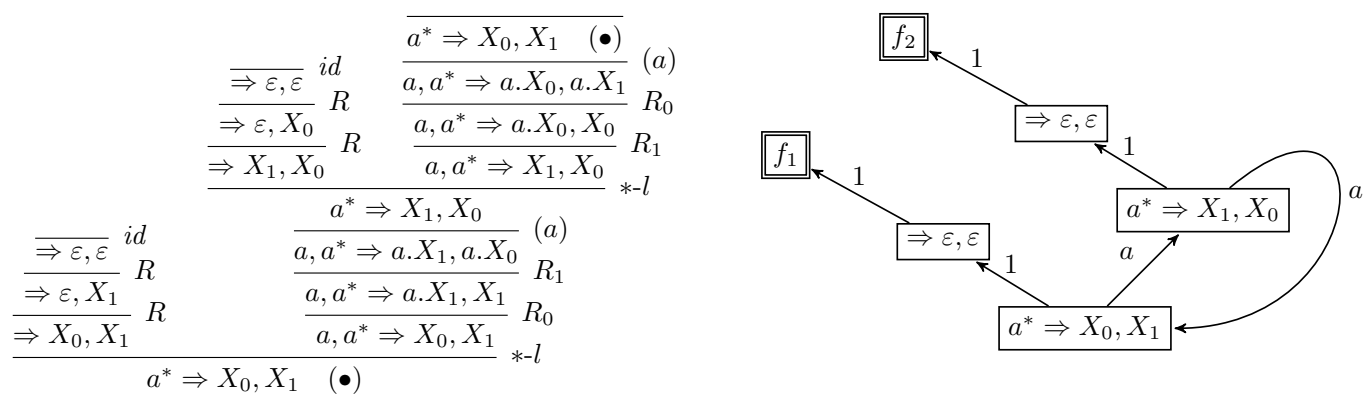

Figure 3: The synchronised proof $\Pi_{0} \times \Pi_{1}$ and its automaton (Ex. 7, 18, 21).

\subsection{Automata from synchronised proofs}

Definition 19 (Automaton of a synchronised proof). Let $\pi$ be a synchronised proof. The automaton of $\pi$ is the NFA $\mathcal{A}(\pi)=\langle Q, T, \iota, F\rangle$, where the set of states is $Q=S \uplus F, S$ being the set of synchronised sequent occurrences of $\pi$ and $F$ being a copy of the conclusions of $\mathcal{H}$ and $i d$ steps, the initial state $\iota$ is the conclusion of $\pi$, and the transition table $T \subseteq Q \times A \cup\{1\} \times Q$ is defined as follows. $(p, l, q) \in T$ if either:

- $q$ is a premiss of $p$ and $l=a$ if the applied rule is a modal rule $(a)$ and $l=1$ otherwise.

- $p=(x \Rightarrow \mathcal{X})($ resp. $p=(\Rightarrow \mathcal{X}))$ is the conclusion of a $\mathcal{H}$ (resp. $i d$ ) rule, $q \in F$ is its copy, and $l=x$ (resp. $l=1)$.

Proviso 20. Since synchronised proofs are represented as finite trees with back-pointers, their automata also have this shape. We call the tree structure underlying automata of this shape simply their trees. In the sequel we suppose that all our NFA have this shape, and that their trees are rooted in the initial state. (Note that every NFA can be put into this form.)

Example 21. The automaton of $\Pi_{0} \times \Pi_{1}$ from Ex. 7, 18 is given on the right of Fig. 3. The accepting states are $f_{1}$ and $f_{2}$, which are the copies of the two occurrences of $\Rightarrow \varepsilon, \varepsilon$. The initial state is $a^{*} \Rightarrow X_{0}, X_{1}$. We omitted states and epsilon transitions for the sake of readability. This automaton recognises the language $a^{*}$, which is the intersection of the languages of $X_{0}$ and $X_{1}$. Note that if we had started with the multiset $X_{0} ; a^{*} c$ in the right-hand side of the sequent from Ex. 7, we would have obtained a similar automaton rather than an automaton for $a^{*} c$ : in general, the constructed automaton recognises the intersection of $\underline{e}^{*} \underline{\Gamma}$ and the $\underline{X}_{1}, \ldots, \underline{X}_{n}$.

We will use some graph theoretic terminology for NFA, which we introduce below.

Definition 22 (Paths, labels, proper loops). Let $\mathcal{A}=\langle Q, T, \iota, F\rangle$ be a NFA and let $l: Q \rightarrow \operatorname{Exp}_{A}$ be a labelling of the states of $\mathcal{A}$.

A path is a sequence $\mathrm{p}=q_{1}, a_{1}, \ldots, q_{k-1}, a_{k_{1}}, q_{k}$ where $q_{i} \in Q$ and $\left\langle q_{i}, a_{i}, q_{i+1}\right\rangle \in T$. It is simple if $q_{i} \neq q_{j}$ when $i \neq j$. We denote by $\operatorname{Simpl}(p, q)$ the set of simple paths from $p$ to $q$. The label of $\mathrm{p}$, denoted $l(\mathrm{p})$ is the sequence $l\left(q_{1}\right), a_{1}, \ldots, l\left(q_{k-1}\right), a_{k_{1}}, l\left(q_{k}\right)$.

A proper loop of $q$ is a sequence $a, \mathrm{p}, b$ s.t. $q, a, \mathrm{p}$ and $\mathrm{p}, b, q$ are simple paths and all states of $\mathrm{p}$ are descendants of $q$ in the tree of $\mathcal{A}$. We write $\operatorname{Loop}(q)$ for the set of proper loops of $q$.

In the following, we denote by $p, q, r, \ldots$ the states of NFA and $\mathrm{p}, \mathrm{q}, \mathrm{r} \ldots$ their paths and loops. 
Remark 23. There are two differences between proper loops and what is usually called a loop: they are not paths in the sense of the previous definition because they do not begin and do not finish by a node. (For that one can complete them by adding nodes to both extremities.) The second difference, more fundamental, is that we do not have the right to visit nodes which are ancestors (in the tree of the automaton) of the node on which we loop.

\subsection{Extracting regular expressions from automata}

Definition 24 (Expression of a NFA). Let $\mathcal{A}=\langle Q, T, \iota, F\rangle$ be a NFA. We define a state labelling function $l: Q \rightarrow \operatorname{Exp}_{A}$ recursively on the tree of $\mathcal{A}$, starting from the leaves. Suppose that we are processing the state $q$, we set:

$$
l(q)=\left(\sum_{\mathrm{p} \in \operatorname{Loop}(q)} l(\mathrm{p})\right)^{*}
$$

(Since leaves cannot have proper loops, they are labelled with $0^{*}$, which is equivalent to 1 in $\ell \mathrm{KA}$; the map $l$ is well defined: thanks to the definition of $\operatorname{Loop}(q)$, we use only the descendants of $q$ in the tree of $\mathcal{A}$ to compute $l(q)$.) The expression $\mathcal{I}(\mathcal{A})$ of $\mathcal{A}$, is defined as follows: ${ }^{1}$

$$
\mathcal{I}(\mathcal{A})=\{l(\mathrm{p}) \mid f \in F, \mathrm{p} \in \operatorname{Simple}(\iota, f)\}
$$

Example 25. We compute the expression of the automaton $\mathcal{A}$ of Ex. 21. For the state labelling $l$, the only state having a proper loop is the initial state. Thus, modulo lKA axioms, we get:

$$
l: q \mapsto \begin{cases}(a a)^{*} & \text { if } q \text { is } a^{*} \Rightarrow X_{0}, X_{1} \\ 1 & \text { otherwise }\end{cases}
$$

There are two paths from the initial state to final states: one going to $f_{1}$ and the other to $f_{2}$. The expression corresponding to the automaton $\mathcal{A}$ is then $\mathcal{I}(\mathcal{A})=(a a)^{*} ;(a a)^{*}, a$. (Again, modulo

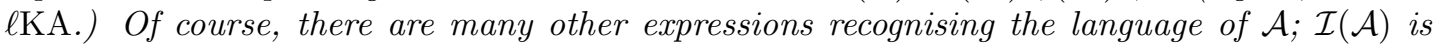
convenient because it is closely related to both $X_{0}$ and $X_{1}$.

Remark 26. We do not state the Kleene theorem (i.e. that $\mathcal{L}(\mathcal{A})=\mathcal{L}(\mathcal{I}(\mathcal{A})$ )) for this construction as we do not need it in the sequel. We point out, however, that it holds.

Turning back to our global argument, we set $\underline{\mathcal{I}} \triangleq \mathcal{I}(\underline{\mathcal{A}})$ where $\underline{\mathcal{A}}$ is the automaton of the synchronous proof $\prod_{i \in[1, n]} \underline{\pi}_{i}$.

\subsection{Regular expressions for arbitrary states}

The computation of $\mathcal{I}(\mathcal{A})$ relies on the precise tree structure of $\mathcal{A}$. We will also need to compute the expression of $\mathcal{A}$ from an arbitrary state $q$ of $\mathcal{A}$, not just the intial state, which we will denote by $\llbracket q \rrbracket$. One could reorganise $\mathcal{A}$ as a tree rooted in $q$, but this would be tedious. Another way to proceed is to use the labelling $l$ of $\mathcal{A}$ (with $\iota$ as the root of $\mathcal{A}$ ), and generalise the computation of $\mathcal{I}(\mathcal{A})$ to arbitrary states. To do so, we need to generalise the notion of simple path:

Definition 27 (Forward path, expression of an arbitrary state). A path $\mathrm{p}=q_{1}, a_{1}, \ldots, a_{k-1}, q_{k}$ in a NFA $\mathcal{A}$ is forward if for every $i<j$ such that $q_{i}=q_{j}$, there is $o$ such that $i<o<j, q_{o} \neq q_{i}$

\footnotetext{
${ }^{1}$ Formally, $\mathcal{I}(\mathcal{A})$ is a multiset; we however interpret it as a single regular expression, as usual in this paper.
} 
and $q_{o}$ is an ancestor of $q_{i}$ in the tree of $\mathcal{A}$. For every state $q$, we write Forward $(q)$ for the set of forward paths from $q$ to a final state, and

$$
\llbracket q \rrbracket_{\mathcal{A}} \triangleq\{l(\mathrm{p}) \mid \mathrm{p} \in \operatorname{Forward}(q)\} .
$$

We write $\llbracket q \rrbracket$ instead of $\llbracket q \rrbracket_{\mathcal{A}}$ when the automaton is clear from the context or when $\mathcal{A}=\underline{\mathcal{A}}$.

We compute forward paths in a concrete graph in App. C; note that they are finitely many, since the number of times any forward path may visit a given node is bounded by the number of nodes between the root and that node.

Remark 28. If $\iota$ is the initial state of $\mathcal{A}$, then a path starting from $\iota$ is forward if and only if it is simple, since $\iota$ is the root of the tree of $\mathcal{A}$. Thus we have that $\mathcal{I}(\mathcal{A})=\llbracket \iota \rrbracket_{\mathcal{A}}$.

Remark 29. If $\pi$ is a synchronised proof and $\mathcal{A}=\mathcal{A}(\pi)$, then $\llbracket x \Rightarrow \mathcal{X} \rrbracket=x$ (resp. $\llbracket \Rightarrow \mathcal{X} \rrbracket=1$ ) if $x \Rightarrow \mathcal{X}$ (resp. $\Rightarrow \mathcal{X})$ is the conclusion of a $\mathcal{H}$ (resp. id) rule.

We do not need to show that $\llbracket q \rrbracket$ is indeed the expression of $\mathcal{A}$ when $q$ is the initial state. Instead, we just need to relate $\llbracket q \rrbracket$ and $\llbracket r \rrbracket$ of two close states $q$ and $r$.

Lemma 30. Let $\mathcal{A}=\langle Q, T, \iota, F\rangle$ be a NFA, $q \in Q$, and $\left\{q_{i}\right\}_{i \in[1, k]},\left\{a_{i}\right\}_{i \in[1, k]}$ be sequences such that $\left\langle q, a_{i}, q_{i}\right\rangle \in T$ for all $i \in[1, k]$. We have $\ell \mathrm{KA}, \mathcal{H} \vdash a_{1} \llbracket q_{1} \rrbracket+\cdots+a_{k} \llbracket q_{k} \rrbracket \leq \llbracket q \rrbracket$, and the following rule is derivable in $\mathrm{HKA}+\mathcal{H}$ :

$$
\frac{\Gamma \rightarrow a_{1} \cdot \llbracket q_{1} \rrbracket ; \ldots ; a_{k} \cdot \llbracket q_{k} \rrbracket}{\Gamma \rightarrow \llbracket q \rrbracket}
$$

Proof. The inequality of $\ell \mathrm{KA}+\mathcal{H}$ follows from derivability of the rule by taking $\Gamma$ to be $a_{1} \llbracket q_{1} \rrbracket+$ $\cdots+a_{k} \llbracket q_{k} \rrbracket$, using Prop. 10 and distributivity. Let us show that the rule is derivable in $\mathrm{HKA}+\mathcal{H}$. For every $i \in[1, k]$, we set $\mathcal{F}_{i}=\left\{q_{i}, \mathrm{p} \mid q, a_{i}, q_{i}, \mathrm{p} \in \operatorname{Forward}(q)\right\}$ the set of paths starting from $q_{i}$ which can be prolonged to forward paths of $q$ by going through $a_{i}$; and we set $L_{i}=\left\{q_{i}, \mathrm{p} \mid a_{i}, q_{i}, \mathrm{p} \in \operatorname{Loop}(q)\right\}$. We first show that $\operatorname{Forward}\left(q_{i}\right) \subseteq \mathcal{F}_{i} \cup\left(L_{i}\right.$, Forward $\left.(q)\right)$. Let $\mathrm{p} \in$ Forward $\left(q_{i}\right)$. There are three cases to distinguish:

- The path $\mathrm{p}$ does not visit $q$. In this case $q, a_{i}, \mathrm{p} \in \operatorname{Forward}(q)$ thus $p \in \mathcal{F}_{i}$.

- $\mathrm{p}$ is of the form $q_{i}, \mathrm{q}, q, \mathrm{r}$ where $\mathrm{q}$ does not contain $q$ but contains an ancestor of $q$. In this case $q, a_{i}, \mathrm{p} \in \operatorname{Forward}(q)$ thus $\mathrm{p} \in \mathcal{F}_{i}$.

- $\mathrm{p}$ is of the form $q_{i}, \mathrm{q}, q, \mathrm{r}$ where $\mathrm{q}$ does not contain $q$ and contains only descendents of $q$. Note that $a_{i}, q_{i}, \mathrm{q}$ is a proper loop of $q$, hence $q_{i}, \mathrm{q} \in L_{i}$ moreover $q, \mathrm{r} \in \operatorname{Forward}(q)$ since the suffix of a forward path is a forward path. We have then $\mathrm{p} \in L_{i}$, Forward $(q)$.

We have that:

$$
\begin{array}{ll}
\left\{a_{i}, l(\mathrm{p}) \mid i \in[1, k], \mathrm{p} \in \mathcal{F}_{i}\right\} & =\cup_{i \in[1, k]} a_{i}, \mathcal{F}_{i} \\
\left\{a_{j}, l(\mathrm{r}), l(q), a_{i}, l(\mathrm{p}) \mid i, j \in[1, k], \mathrm{p} \in \mathcal{F}_{i}, \mathrm{r} \in L_{j}\right\} & =\cup_{i \in[1, k]} a_{i}, L_{i}, \text { Forward }(q)
\end{array}
$$

Thus we get the following derivation:

$$
\frac{\Gamma \rightarrow\left\{a_{i}, l(\mathrm{p}) \mid i \in[1, k], \mathrm{p} \in \mathcal{F}_{i}\right\} \cup\left\{a_{j}, l(\mathrm{r}), l(q), a_{i}, l(\mathrm{p}) \mid i, j \in[1, k], \mathrm{p} \in \mathcal{F}_{i}, \mathrm{r} \in L_{j}\right\}}{\frac{\Gamma \rightarrow\left\{l(q), a_{i}, l(\mathrm{p}) \mid i \in[1, k], \mathrm{p} \in \mathcal{F}_{i}\right\}}{\Gamma \rightarrow \llbracket q \rrbracket} w k} *-r,+-r, w k
$$

We conclude using weakening $(w k)$. 


\subsection{A minorant lemma}

We conclude this section by a generic lemma which we use twice in the proofs of the next section. This lemma states that the expression of a state $q, \llbracket q \rrbracket_{\mathcal{A}}$, is provably smaller (in $\ell \mathrm{KA}+\mathcal{H}$ ) than the value of any compatible function at $q$. Let us define first this notion of compatibility.

Definition 31. Let $\mathcal{A}=\langle Q, T, \iota, F\rangle$ be a NFA and $f: Q \rightarrow \operatorname{Exp}_{A}$ a function from states to expressions. We say that $f$ is compatible with $\mathcal{A}$ if $\ell \mathrm{KA}, \mathcal{H} \vdash 1 \leq f(q)$ whenever $q$ is a leaf of $\mathcal{A}$ and for all transitions $\langle q, a, r\rangle \in T$, we have $\ell \mathrm{KA}, \mathcal{H} \vdash a f(r) \leq f(q)$.

For instance, the function $\llbracket \cdot \rrbracket_{\mathcal{A}}: q \mapsto \llbracket q \rrbracket_{\mathcal{A}}$ is compatible with $\mathcal{A}$ thanks to Lem. 30 .

Lemma 32 (Minorant lemma). Let $f$ be compatible with a NFA $\mathcal{A}$; for each state q, we have:

$$
\ell \mathrm{KA}, \mathcal{H} \vdash \llbracket q \rrbracket_{\mathcal{A}} \leq f(q)
$$

Proof. Since $\llbracket q \rrbracket_{\mathcal{A}}=\sum_{\mathrm{p} \in \text { Forward }(q)} l(\mathrm{p})$, we should show that for any path $\mathrm{p} \in \operatorname{Forward}(q)$, $\ell \mathrm{KA}, \mathcal{H} \vdash l(\mathrm{p}) \leq f(q)$. We proceed by induction on the length of the path $\mathrm{p}$. We can write $\mathrm{p}=q, a, r, \mathrm{q}$ where $r$ is a child of $q$ in $\mathcal{A}$. Since $l(\mathrm{p})=l(q) a l(r \mathrm{q})$, it is enough to show that $\ell \mathrm{KA}, \mathcal{H} \vdash l(q) f(q) \leq f(q)$ and $\ell \mathrm{KA}, \mathcal{H} \vdash a l(r \mathrm{q}) \leq f(q)$. Let us show the second inequality first; we will show the first inequality afterwards, out of the induction. Note that $r q \in$ Forward $(r)$ thus $l(r q) \leq f(r)$ by induction, and then $a l(r q) \leq a f(r)$. By compatibility of $f$ we have that $a f(r) \leq f(q)$ which concludes the proof.

Now, let us show now that $\ell \mathrm{KA}, \mathcal{H} \vdash l(q) f(q) \leq f(q)$. We proceed by induction on $q$, starting from the leaves of the tree of $\mathcal{A}$. Recall that $l(q)=S^{*}$ where $S=\sum_{\mathrm{p} \in \operatorname{Loop}(q)} l(\mathrm{p})$. By the implication (L) of $\ell \mathrm{KA}$, it suffices to show that $\ell \mathrm{KA}, \mathcal{H} \vdash S f(q) \leq f(q)$, or equivalently that for each path $\mathrm{p} \in \operatorname{Loop}(q), \ell \mathrm{KA}, \mathcal{H} \vdash l(\mathrm{p}) f(q) \leq f(q)$.

Let $\mathrm{p}=a_{0}, q_{1}, \ldots, q_{k}, a_{k} \in \operatorname{Loop}(q)$. We set $q_{0} \triangleq q$ and $q_{k+1} \triangleq q$. For every $i \in[1, k]$, we set $\mathrm{s}_{i} \triangleq a_{i}, q_{i+1} \ldots q_{k}, a_{k}$ and $\mathrm{t}_{i} \triangleq q_{i}, a_{i} \ldots q_{k}, a_{k}$. We have that:

$$
l\left(\mathrm{~s}_{i}\right)=a_{i} l\left(\mathrm{t}_{i+1}\right) \quad \text { and } \quad l\left(\mathrm{t}_{i+1}\right)=l\left(q_{i+1}\right) l\left(\mathrm{~s}_{i+1}\right)
$$

We show by an internal induction on the length of $s_{i}$ that:

$$
\ell \mathrm{KA}, \mathcal{H} \vdash l\left(\mathrm{~s}_{i}\right) f(q) \leq f\left(q_{i}\right)
$$

By compatibility of $f$, we have that $\ell \mathrm{KA}, \mathcal{H} \vdash a_{i} f\left(q_{i+1}\right) \leq f\left(q_{i}\right)$, so it is enough to show that $l\left(\mathrm{~s}_{i}\right) f(q) \leq a_{i} f\left(q_{i+1}\right)$ or, deleting $a_{i}$ from both sides, that $l\left(\mathrm{t}_{i+1}\right) f(q) \leq f\left(q_{i+1}\right)$. For that, we show that $l\left(q_{i+1}\right) f\left(q_{i+1}\right) \leq f\left(q_{i+1}\right)$ and $l\left(\mathrm{~s}_{i+1}\right) f(q) \leq f\left(q_{i+1}\right)$. The first holds thanks to the external induction on $q$, while the second one comes from the internal induction.

\section{Deriving the inequalities for the invariant}

\section{$5.1 \quad$ Deriving $\ell \mathrm{KA}, \mathcal{H} \vdash \underline{\Gamma} \leq \underline{\mathcal{I}}$}

Let us show the first inequality that should be satisfied by the invariant:

Theorem 33. $\ell \mathrm{KA}, \mathcal{H} \vdash \underline{\Gamma} \leq \underline{\mathcal{I}}$

Since $\# \underline{\Gamma}<\#\left(\underline{e}^{*}, \underline{\Gamma}\right)$, this follows from the global induction hypothesis and:

Proposition 34. HKA, $\mathcal{H} \stackrel{\omega}{ }^{\Gamma} \underline{\Gamma} \rightarrow \underline{\mathcal{I}}$. 
We generalise the statement into the following lemma.

Lemma 35. Let $\pi$ be a synchronised proof of $\Gamma \Rightarrow \mathcal{X}$. We have $\mathrm{HKA}, \mathcal{H} \vdash^{\omega} \Gamma \rightarrow \llbracket \Gamma \Rightarrow \mathcal{X} \rrbracket_{\mathcal{A}(\pi)}$.

Prop. 34 follows from Lem. 35 by considering $\underline{\pi}$ and by recalling that $\underline{\mathcal{I}}=\llbracket \underline{\Gamma} \Rightarrow \underline{X}_{1}, \ldots, \underline{X}_{n} \rrbracket$ as noticed in Rmk. 28.

Proof of Lem. 35. Let $\mathcal{A}=\mathcal{A}(\pi)$; we build the desired proof $\theta(\pi)$ coinductively, by case analysis on the last rule $\rho$ applied in $\pi$.

- $\rho$ is a left rule:

$$
\text { If } \pi=\frac{\left\{\frac{\pi_{i}}{\Gamma_{i} \Rightarrow \mathcal{X}}\right\}_{i \in I}}{\Gamma \Rightarrow \mathcal{X}} 1 \quad \text { then } \quad \theta(\pi)=\frac{\left\{\frac{\frac{\theta\left(\pi_{i}\right)}{\Gamma_{i} \rightarrow \llbracket \Gamma_{i} \Rightarrow \mathcal{X} \rrbracket_{\mathcal{A}}}}{\Gamma_{i} \rightarrow \bigcup_{j \in I} \llbracket \Gamma_{j} \Rightarrow \mathcal{X} \rrbracket_{\mathcal{A}}} w k\right\}_{i \in I} 1}{\frac{\Gamma \rightarrow \bigcup_{j \in I} \llbracket \Gamma_{j} \Rightarrow \mathcal{X} \rrbracket_{\mathcal{A}}}{\Gamma \rightarrow \llbracket \Gamma \Rightarrow \mathcal{X} \rrbracket_{\mathcal{A}}}\left(\text { Lem. } 30^{\dagger}\right)}
$$

(†) $\left\{\Gamma_{i} \Rightarrow \mathcal{X}\right\}_{i \in I}$ are the children of $\Gamma \Rightarrow \mathcal{X}$; the corresponding transitions are labelled with 1.

- $\rho$ is a right rule:

$$
\text { If } \quad \pi=\frac{\frac{\pi^{\prime}}{\Gamma \Rightarrow \mathcal{X}, Z, \mathcal{Y}}}{\Gamma \Rightarrow \mathcal{X}, Y, \mathcal{Y}} \mathrm{r} \quad \text { then } \quad \theta(\pi)=\frac{\frac{\theta\left(\pi^{\prime}\right)}{\Gamma \rightarrow \llbracket \Gamma \Rightarrow \mathcal{X}, Z, \mathcal{Y} \rrbracket_{\mathcal{A}}}}{\Gamma \rightarrow \llbracket \Gamma \Rightarrow \mathcal{X}, Y, \mathcal{Y} \rrbracket_{\mathcal{A}}} \text { (Lem. 30) }
$$

- $\rho$ is the hypothesis rule: we use the fact that $\llbracket x \Rightarrow \mathcal{X} \rrbracket_{\mathcal{A}}=x$, as noticed in Rmk. 29 .

$$
\text { If } \quad \pi=\overline{x \Rightarrow \mathcal{X}} \mathcal{H} \quad \text { then } \quad \theta(\pi)=\overline{x \rightarrow \llbracket x \Rightarrow \mathcal{X} \rrbracket_{\mathcal{A}}} \mathcal{H}
$$

- Similarly when $\rho$ is the identity rule.

- $\rho$ is the modal rule $(a)$.

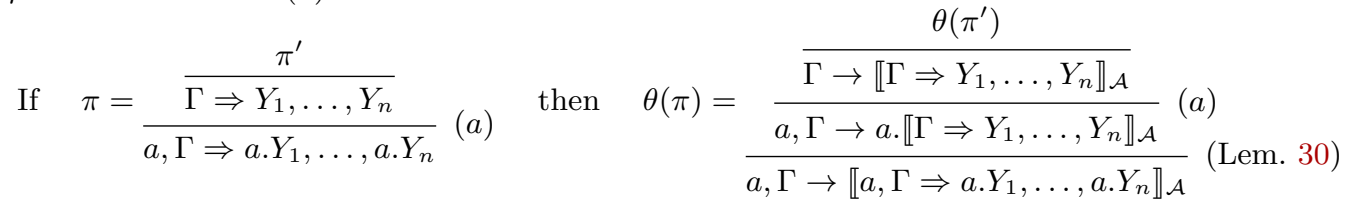

The obtained HKA $+\mathcal{H}$ preproof is clearly regular and fair for $*-l$.

\subsection{Deriving $\ell \mathrm{KA}, \mathcal{H} \vdash \underline{e}, \underline{\mathcal{I}} \leq \underline{\mathcal{I}}$}

We now show that $\mathcal{I}$ is stable by composition with $e$ on the left. This is the part of the proof that justifies the introduction of hypotheses.

Theorem 36. $\ell \mathrm{KA}, \mathcal{H} \vdash \underline{e}, \underline{\mathcal{I}} \leq \underline{\mathcal{I}}$.

Notation 37. If $\mathcal{Y}=Y_{1}, \ldots, Y_{n}$ and $\sigma:[1, n] \rightarrow[1, n]$ we write $\mathcal{Y}_{\sigma}$ for the $n$-uple $Y_{\sigma(1)}, \ldots, Y_{\sigma(n)}$.

Let $\underline{x}$ be a fresh letter (not appearing in $\underline{e}, \underline{\mathcal{I}}$ nor in $\mathcal{H}$ ), and let $\mathcal{H}_{\underline{x}}$ be the following set of hypotheses, where $\underline{\mathcal{X}} \triangleq \underline{X}_{1}, \ldots, \underline{X}_{n}$ :

$$
\mathcal{H}_{\underline{x}} \triangleq\left\{\underline{x} \leq \llbracket \underline{e}^{*}, \underline{\Gamma} \Rightarrow \underline{\mathcal{X}}_{\sigma} \rrbracket \mid \sigma:[1, n] \rightarrow[1, n]\right\}
$$


To prove Thm. 36, we first show that under these new hypotheses about $\underline{x}$, the sequent $\underline{e}, \underline{x} \rightarrow \underline{\mathcal{I}}$ is provable in HKA $+\mathcal{H}+\mathcal{H}_{x}$ (Prop. 38 below). Intuitively, we do so by mimicking the proof of $\underline{e}, \underline{e}^{*}, \underline{\Gamma} \rightarrow \underline{X}_{1}$ : when we reach a sequent of the form $\underline{e}^{*}, \underline{\Gamma} \rightarrow \underline{X}_{i}$ in the latter, we reach a sequent of the form $\underline{x} \rightarrow \llbracket \underline{e}^{*}, \underline{\Gamma} \Rightarrow \underline{\mathcal{X}}_{\sigma} \rrbracket$ in the former, which can be closed using a hypothesis from $\mathcal{H}_{\underline{x}}$.

Once we get this $\mathrm{HKA}+\mathcal{H}+\mathcal{H}_{\underline{x}}$ proof of $\underline{e}, \underline{x} \rightarrow \underline{\mathcal{I}}$, we translate it into a $\ell \mathrm{KA}+\mathcal{H}+\mathcal{H}_{\underline{x}}$ proof using the induction hypothesis, since $\#(\underline{e}, \underline{x})<\#\left(\underline{e}, \underline{e}^{*}, \underline{\Gamma}\right)$. To get rid of the variable $\underline{x}$ and the hypotheses about it, we show that $\underline{\mathcal{I}}$ satisfies all the hypotheses about $\underline{x}$ (Prop. 40 below), thus $\underline{x}$ can be safely replaced by $\mathcal{I}$, and the hypotheses $\mathcal{H}_{\underline{x}}$ that were introduced to characterise $\underline{x}$ are not needed anymore. Since $\underline{x}$ does not appear in $\underline{\underline{e}}, \underline{\Gamma}$, and $\underline{\mathcal{I}}$, we obtain Thm. 36 .

We now show Props. 38 and 40. As before, we first need to generalise their statements.

Proposition 38. HKA, $\mathcal{H}, \mathcal{H}_{\underline{x}} \vdash^{\omega} \underline{e}, \underline{x} \rightarrow \underline{\mathcal{I}}$.

Lemma 39. Let $\delta$ be a subproof of $\underline{\pi}$ rooted at $\Delta, \underline{e}^{*}, \underline{\Gamma} \Rightarrow \mathcal{Y}$. We have that $\mathrm{HKA}, \mathcal{H}, \mathcal{H}_{\underline{x}} \vdash^{\omega}$ $\Delta, \underline{x} \rightarrow \llbracket \Delta, \underline{e}^{*}, \underline{\Gamma} \Rightarrow \mathcal{Y} \rrbracket$.

Proof. We proceed in the exact same way as for Lem. 35, that is by building the desired proof $\theta(\delta)$ coinductively, by case analysis of the last rule applied in $\delta$ and by mimicking this rule in HKA $+\mathcal{H}+\mathcal{H}_{\underline{x}}$ using Lem. 30. The only new case is when the conclusion of $\delta$ is $\underline{e}^{*}, \underline{\Gamma} \Rightarrow \mathcal{Y}$ (that is, $\Delta=\varnothing)$. By construction of $\underline{\pi}$, we know that $\mathcal{Y}=\underline{\mathcal{X}}_{\sigma}$ for some $\sigma:[1, n] \rightarrow[1, n]$. The proof $\theta(\delta)$ in this case is just an application of a hypothesis from $\mathcal{H}_{\underline{x}}$.

Prop. 38 now follows by the following argument: since every $\underline{\pi}_{i}$ ends with an application of $*-l$ rule to $\underline{e}^{*}, \underline{\Gamma} \rightarrow \underline{X}_{i}$, we have that $\underline{\pi}$ has the form on the left below. Recall that $\underline{\mathcal{I}}=\llbracket \underline{e}^{*}, \underline{\Gamma} \Rightarrow \underline{\mathcal{X}} \rrbracket$, so we get the $\mathrm{HKA}+\mathcal{H}+\mathcal{H}_{\underline{x}}$ proof on the right.

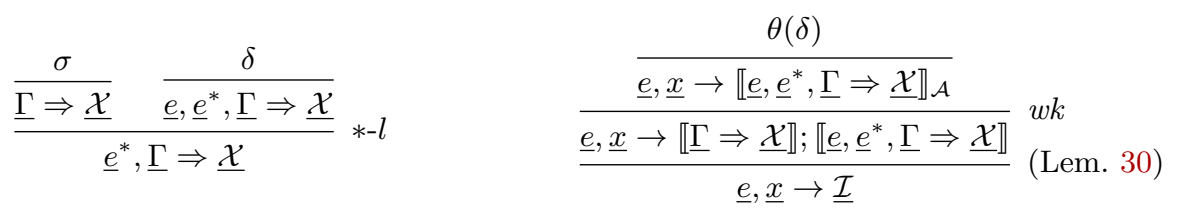

Proposition 40. For every $\sigma:[1, n] \rightarrow[1, n]$, we have $\ell \mathrm{KA}, \mathcal{H} \vdash \underline{\mathcal{I}} \leq \llbracket \underline{e}^{*}, \underline{\Gamma} \leq \underline{\mathcal{X}}_{\sigma} \rrbracket$.

Proof. If $q=(\Delta \Rightarrow \mathcal{Y})$ is a state of $\underline{\mathcal{A}}$, we write $q_{\sigma}$ for the state $\Delta \Rightarrow \mathcal{Y}_{\sigma}$.

Using this notation, and recalling that $\underline{\mathcal{I}}=\llbracket \underline{\iota} \rrbracket$, we can rephrase the statement as $\ell \mathrm{KA}, \mathcal{H} \vdash$ $\llbracket \underline{\iota} \leq \llbracket \underline{\iota} \sigma_{\sigma} \rrbracket$. We show the following generalisation: for every state $q$ of $\underline{\mathcal{A}}$, we have $\ell \mathrm{KA}, \mathcal{H} \vdash \llbracket q \rrbracket \leq$ $\llbracket q_{\sigma} \rrbracket$. This is a consequence of Lem. 32, when we consider the function $f: q \mapsto \llbracket q_{\sigma} \rrbracket$. Indeed, $f$ is compatible, since when $\langle q, a, r\rangle \in \underline{T}$, we have also that $\left\langle q_{\sigma}, a, r_{\sigma}\right\rangle \in \underline{T}$, thus by Lem. 30 we have that $\ell \mathrm{KA}, \mathcal{H} \vdash a \llbracket r_{\sigma} \rrbracket \leq \llbracket q_{\sigma} \rrbracket$, which concludes the proof.

\subsection{Deriving $\ell \mathrm{KA}, \mathcal{H} \vdash \underline{\mathcal{I}} \leq X_{1}$}

We finally prove the third requirement for the invariant:

Theorem 41. $\ell \mathrm{KA}, \mathcal{H} \vdash \underline{\mathcal{I}} \leq \underline{X}_{1}$.

Proof. Given a state $q$ of a NFA $\mathcal{A}$, if $q=\Delta \Rightarrow Y_{1}, \ldots, Y_{n}$ we set $\pi_{1}(q)=Y_{1}$; if $q$ is a final state, we set $\pi_{1}(q)=1$. We can write Thm. 41 using this notation as $\ell \mathrm{KA}, \mathcal{H} \vdash \llbracket \underline{\iota} \leq \leq \pi_{1}(\underline{\iota})$. We show the following generalisation: for every state $q$ of $\mathcal{A}$, we have $\ell \mathrm{KA}, \mathcal{H} \vdash \llbracket q \rrbracket \leq \pi_{1}(q)$. This is a consequence of Lem. 32, when we consider the function $f: q \mapsto \pi_{1}(q)$. Indeed, by analysing all the synchronised rules, it is easy to show that $\ell \mathrm{KA}, \mathcal{H} \vdash a \pi_{1}(r) \leq \pi_{1}(q)$, whenever $\langle q, a, r\rangle$ is a transition of $\underline{\mathcal{A}}$, thus $f$ is compatible.

This concludes our proof of left-handed completeness (Thm. 2). 


\section{Conclusion}

We have given a new and direct proof of completeness for left-handed Kleene algebra with respect to rational language inclusions. The left-handed derivations we construct closely follow the cyclic proofs of HKA, whose structure in turn corresponds to standard coinductive algorithms for comparing regular expressions. We use the implication of $\ell \mathrm{KA}(\mathrm{L})$ at two places: when a starred sub-expression of the left-hand side is unfolded, through the invariant lemma (Lem. 3), and to prove the the minorant lemma (Lem. 32, which is used twice to show that the constructed invariants fulfil the requirements of the invariant lemma).

Unlike Krob[26], Boffa [7], or Silva and Kozen [23], we do not need to exploit any specific laws about Kleene star, like Conway's axioms C11 and C12 ('sumstar' and 'productstar'). Our proof implies that they already follow from (L) and (L') in idempotent semirings (see App. B).

From a metalogical perspective, this work constitutes an example of translating cyclic proofs to ones that are 'inductive', a matter of considerable interest recently, cf. [29, 15, 1, 6, 13]. While the converse direction is, in general, routine (see, e.g., [10]), the technicalities encountered in this work contribute to our understanding of when the two proof-theoretic phenomena are equipotent. We point out that, despite the aforementioned recent breakthroughs in simulating cyclicity via induction, there are several settings where they do not coincide, e.g. [5].

We conclude with a summary of the complete axiomatisations known so far. Below, KA stands for Kleene algebra, $r$ KA stands for right-handed Kleene algebra (the symmetric version of left-handed Kleene algebra), CA stands for 'Conway algebra', i.e. those algebras satisfying Conway's axioms, proven complete by Krob [26], and BA stands for 'Boffa algebra' [8], which is a symmetric axiomatisation where the only implication is $e e=e \Rightarrow e^{*}=1+e$.

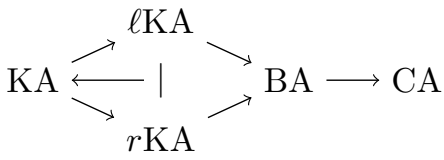

Every left (or right) handed Kleene algebra is a Boffa algebra [8], and every Boffa algebra is a Conway algebra [7]. The converse implications do not hold. There is a Conway algebra which is not even a Boffa algebra [23] (even a finite one); Ex. 1 shows that there are left-handed Kleene algebras which are not right-handed (and vice-versa by symmetry); and one can find Boffa algebras which are neither left nor right handed - see App. G. Note however that the four classes on the left coincide on finite structures: every finite Boffa algebra is a Kleene algebra-App. E.

Krob's completeness result [26] ensures once and for all that the five axiomatisations are complete for rational language inclusions. Much simpler proofs have been given for KA in [20] and $\ell \mathrm{KA}$ in [23] and the present work, and thus $r \mathrm{KA}$ by symmetry. Whether relying on Krob's extensive proof can be avoided for BA remains open: the techniques developed in the present paper do not seem to apply to such structures. 


\section{A Idempotent semirings}

We list here the axioms of idempotent semirings, for the sake of self-containedness.

$$
\begin{gathered}
e+(f+g)=(e+f)+g \quad e+f=f+e \quad r+e=e \quad e+0=e \\
e \cdot(f \cdot g)=(e \cdot f) \cdot g \quad e \cdot 1=e=1 \cdot e \\
e \cdot(f+g)=e \cdot f+e \cdot g \quad(e+f) \cdot g=e \cdot g+f \cdot g \quad e \cdot 0=0=0 \cdot e
\end{gathered}
$$

We also recall that in such semirings, one gets a partial order by setting $x \leq y$ if $x+y=y$. Sum acts as a supremum operation for this partial order: $x+y \leq z$ iff $x \leq z$ and $y \leq z, 0$ is a bottom element, and product is monotone.

\section{B More HKA proofs}

We give some examples of HKA proofs, namely of Conway's axioms C11 and C12 [12, p. 25]:

$$
\text { (C11) }(a+b)^{*}=a^{*}\left(b a^{*}\right)^{*}
$$

$$
(a b)^{*}=1+a(b a)^{*} b
$$

(C11) left to right:

$$
\begin{aligned}
& \overline{(a+b)^{*} \rightarrow a^{*},\left(b a^{*}\right)^{*}(\bullet)} \\
& \overline{b,(a+b)^{*} \rightarrow b, a^{*},\left(b a^{*}\right)^{*}}(b) \\
& \overline{b,(a+b)^{*} \rightarrow \varepsilon ; b, a^{*},\left(b a^{*}\right)^{*}} w k
\end{aligned}
$$

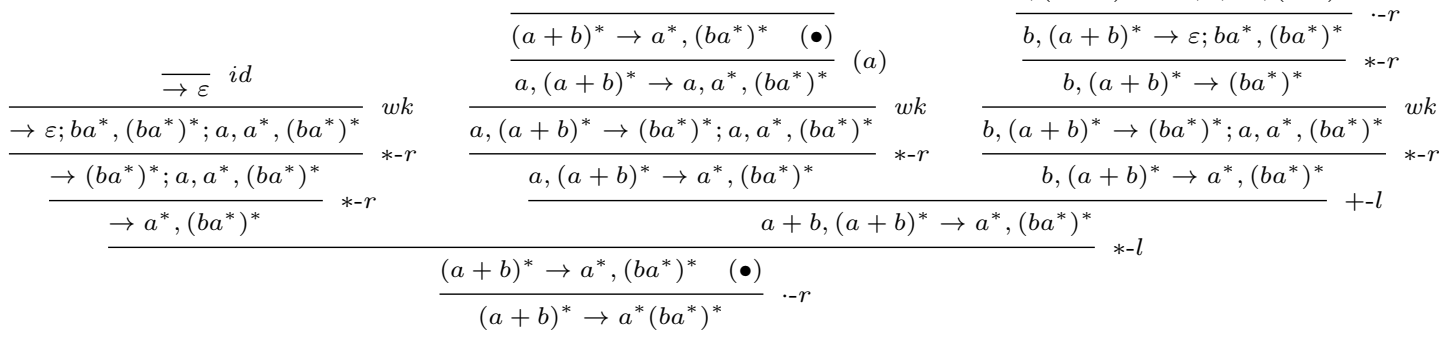

(C11) right to left:

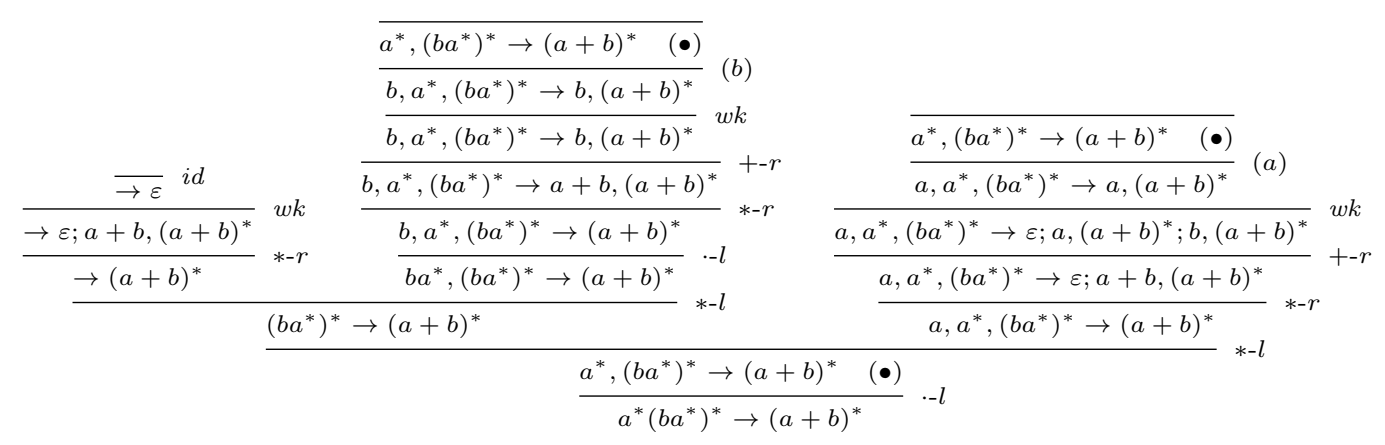


(C12) left to right:

$$
\begin{aligned}
& \overline{(a b)^{*} \rightarrow \varepsilon ; a,(b a)^{*}, b \quad(\bullet)} \\
& \overline{b,(a b)^{*} \rightarrow b ; b, a,(b a)^{*}, b}(b) \\
& {\overline{\frac{b,(a b)^{*} \rightarrow b ; b a,(b a)^{*}, b}{b,(a b)^{*} \rightarrow(b a)^{*}, b}}}_{*-r}^{\cdot-r}
\end{aligned}
$$

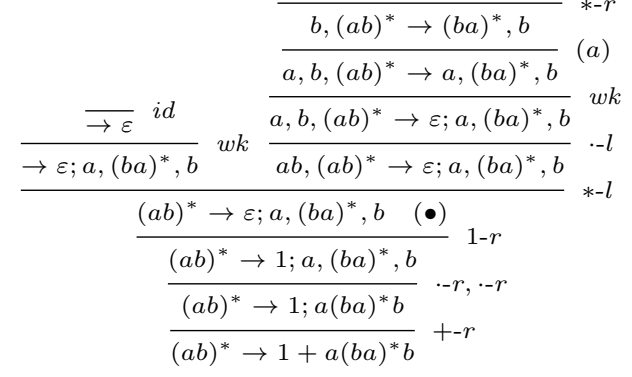

(C12) right to left:

(Note that we could use a back-pointer earlier in the proof on the right, just after the top-right modal (b)-step, but this back-pointer would not point to a $*-l$ step.)

Unlike the proof from Ex. 7, these four proofs are easy to translate to $\ell \mathrm{KA}$ : all their $*-l$ steps satisfy the simplifying assumption $(\star)$ from Sect. 3 so that we do not need to compute complicated invariants: their (unique) succedent does the job.

\section{Examples and non-examples of forward paths}

In the table below, we compute $l(\cdot)$ (Def. 24) and the forward paths (Def. 27) of the graph on the right. We have underlined the nodes that permit loops in forward paths: in the path $1 b 2 e 4 f \underline{0} a 1 c 3$, the node 0 makes it possible to visit 1 twice; in the path $2 d \underline{1} b 2 e 4 f \underline{0} a 1 c 3$, the node 1 makes it possible to visit 2 twice, and the node 0 makes it possible to visit 1 twice.

The paths $1 b 2 d 1 b 2 e 4 g 5$ and $2 e 4 f 0 a 1 b 2 d 1 c 3$ are not forward: there is no node permitting to visit 1 twice. (Intuitively, these paths are already covered by the labels of the forward paths $1 b 2 e 4 g 5$ and $2 e 4 f 0 a 1 c 3$, since $l(1)=(b d)^{*}$.)

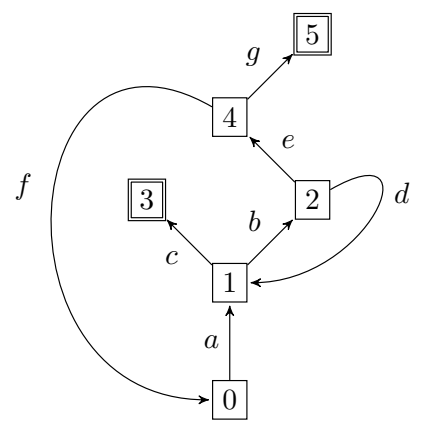

\begin{tabular}{c|c|c}
$n$ & $l(n)$ & Forward $(n)$ \\
\hline 0 & $\left(a(b d)^{*} b e f\right)^{*}$ & $0 a 1 c 3,0 a 1 b 2 e 4 g 5$ \\
1 & $(b d)^{*}$ & $1 c 3,1 b 2 e 4 f \underline{0} a 1 c 3,1 b 2 e 4 g 5,1 b 2 e 4 f \underline{0} a 1 b 2 e 4 g 5$ \\
2 & 1 & $2 d 1 c 3,2 e 4 f 0 a 1 c 3,2 d \underline{1} b 2 e 4 f \underline{0} a 1 c 3,2 e 4 g 5,2 e 4 f \underline{0} a 1 b 2 e 4 g 5$ \\
3 & 1 & 3 \\
4 & 1 & $4 f 0 a 1 c 3,4 g 5,4 f \underline{0} a 1 b 2 e 4 g 5$ \\
5 & 1 & 5
\end{tabular}

\section{Counter-example to the converse of Thm. 14}

It suffices to consider a hypothesis $a \leq b$, and the sequent $a c \rightarrow b c$. We immediately get $\ell \mathrm{KA}, a \leq b \vdash a c \leq b c$, but HKA, $a \leq b \nvdash^{\psi} a c \rightarrow b c$. Whether one can find a cut-free variant of $\mathrm{HKA}+\mathcal{H}$ to capture derivability in $\ell \mathrm{KA}+\mathcal{H}$ remains open; decidability is also open. 


\section{E Every finite Boffa algebra is a Kleene algebra}

Let $x$ be an element of a finite Boffa algebra; we show that $x^{*}=X$, where $X$ is the (necessarily finite) sum of all powers of $x: X:=\Sigma_{i} x^{i}$. First, $x^{*}$ is above all powers of $x$, so that $X \leq x^{*}$. Then, by distributivity, we have $X X=X$, so that $X^{*}=1+X=X$ by Boffa's implication. Since $x \leq X$, we conclude $x^{*} \leq X^{*}=X$ by monotonicity. The two implications (L) and (R) of Kleene algebra follow from distributivity and this characterisation of Kleene star.

\section{F A left-but-not-right handed Kleene algebra}

We show that the construction from Ex. 1 may yield left-handed Kleene algebras which are not right-handed. Consider the complete lattice of extended natural numbers, $\mathbb{N} \cup\{\infty\}$, under the usual order $<$. Since $<$ is total on $\mathbb{N} \cup\{\infty\}$, monotone functions preserve finite suprema. Take the following monotone functions: $g(i)=0$ and $f(i)=i+1$ if $i<\infty$, and $f(\infty)=g(\infty)=\infty$. We have $g+g \cdot f \leq g$, but $g \cdot f^{*} \not \leq g:\left(g \cdot f^{*}\right)(0)=g\left(f^{*}(0)\right)=g(\infty)=\infty$, while $g(0)=0$.

\section{G A Boffa algebra which is neither left nor right handed}

We take natural numbers ordered in the usual sense, completed with three elements $\perp, \omega$ and $T$ such that $\perp<i<\omega<\top$ for all $i \in \mathbb{N}$. We define a commutative Boffa algebra as follows; the bottom element (0) of the algebra is $\perp$ and the identity element (1) is 0 .

\begin{tabular}{c|cccc}
+ & $\perp$ & $j$ & $\omega$ & $\top$ \\
\hline$\perp$ & $\perp$ & $j$ & $\omega$ & $\top$ \\
$i$ & $i$ & $\max (i, j)$ & $\omega$ & $\top$ \\
$\omega$ & $\omega$ & $\omega$ & $\omega$ & $\top$ \\
$\top$ & $\top$ & $\top$ & $\top$ & $\top$
\end{tabular}

\begin{tabular}{c|cccc}
$\cdot$ & $\perp$ & $j$ & $\omega$ & $\top$ \\
\hline$\perp$ & $\perp$ & $\perp$ & $\perp$ & $\perp$ \\
$i$ & $\perp$ & $i+j$ & $\omega$ & $\top$ \\
$\omega$ & $\perp$ & $\omega$ & $\top$ & $\top$ \\
$\top$ & $\perp$ & $\top$ & $\top$ & $\top$
\end{tabular}

\begin{tabular}{c|c}
$*$ & \\
\hline$\perp$ & 0 \\
0 & 0 \\
$i>0$ & $\omega$ \\
$\omega$ & $\top$ \\
$\top$ & $\top$
\end{tabular}

The idempotent elements are $\perp, 0$ and $\top$; $\omega$ is not idempotent so that the equality $\omega^{*}=\top$ does not break Boffa's implication. This algebra is not left-handed (and thus not right-handed since it is commutative): for $x=z=\omega$ and $y=1$, we have $z+y x=\omega=x$ but $y^{*} z=\top \not \leq x$.

\section{References}

[1] B. Afshari and G. E. Leigh. Cut-free completeness for modal mu-calculus. In Proc. LiCS, pages $1-12,2017$.

[2] C. J. Anderson, N. Foster, A. Guha, J.-B. Jeannin, D. Kozen, C. Schlesinger, and D. Walker. NetKAT: semantic foundations for networks. In Proc. POPL, pages 113-126. ACM, 2014.

[3] A. Angus and D. Kozen. Kleene algebra with tests and program schematology. Technical Report TR2001-1844, CS Dpt., Cornell University, July 2001.

[4] V. M. Antimirov. Partial derivatives of regular expressions and finite automaton constructions. Theoretical Computer Science, 155(2):291-319, 1996.

[5] S. Berardi and M. Tatsuta. Classical system of martin-löf's inductive definitions is not equivalent to cyclic proof system. In Proc. FoSSaCS, pages 301-317, 2017.

[6] S. Berardi and M. Tatsuta. Equivalence of inductive definitions and cyclic proofs under arithmetic. In Proc. LiCS, pages 1-12, 2017. 
[7] M. Boffa. Une remarque sur les systèmes complets d'identités rationnelles. Informatique Théorique et Applications, 24:419-428, 1990.

[8] M. Boffa. Une condition impliquant toutes les identités rationnelles. Informatique Théorique et Applications, 29(6):515-518, 1995.

[9] T. Braibant and D. Pous. An efficient Coq tactic for deciding Kleene algebras. In Proc. 1st ITP, volume 6172 of Lecture Notes in Computer Science, pages 163-178. Springer Verlag, 2010.

[10] J. Brotherston and A. Simpson. Sequent calculi for induction and infinite descent. Journal of Logic and Computation, 21(6):1177-1216, 2011.

[11] J. A. Brzozowski. Derivatives of regular expressions. Journal of the ACM, 11(4):481-494, 1964.

[12] J. H. Conway. Regular algebra and finite machines. Chapman and Hall, 1971.

[13] A. Das. On the logical complexity of cyclic arithmetic. CoRR, abs/1807.10248, 2018.

[14] A. Das and D. Pous. A cut-free cyclic proof system for Kleene algebra. In Proc. TABLEAUX, volume 10501 of Lecture Notes in Computer Science, pages 261-277. Springer Verlag, 2017.

[15] A. Doumane. Constructive completeness for the linear-time $\mu$-calculus. In Proc. LiCS, pages 1-12, 2017.

[16] C. A. R. Hoare, B. Möller, G. Struth, and I. Wehrman. Concurrent Kleene Algebra. In Proc. CONCUR, volume 5710 of Lecture Notes in Computer Science, pages 399-414. Springer Verlag, 2009.

[17] S. C. Kleene. Representation of events in nerve nets and finite automata. In Automata Studies, pages 3-41. Princeton University Press, 1956.

[18] L. Kot and D. Kozen. Kleene algebra and bytecode verification. Electronic Notes in Theoretical Computer Science, 141(1):221-236, 2005.

[19] D. Kozen. A completeness theorem for Kleene algebras and the algebra of regular events. In Proc. LiCS, pages 214-225. IEEE, 1991.

[20] D. Kozen. A completeness theorem for Kleene algebras and the algebra of regular events. Information and Computation, 110(2):366-390, 1994.

[21] D. Kozen. On Hoare logic and Kleene algebra with tests. ACM Trans. Comput. Log., 1(1):60-76, 2000.

[22] D. Kozen and M.-C. Patron. Certification of compiler optimizations using Kleene algebra with tests. In Proc. CL2000, volume 1861 of Lecture Notes in Artificial Intelligence, pages 568-582. Springer Verlag, 2000.

[23] D. Kozen and A. Silva. Left-handed completeness. In Proc. RAMiCS, volume 7560 of Lecture Notes in Computer Science, pages 162-178. Springer Verlag, 2012.

[24] D. Kozen and J. Tiuryn. Substructural logic and partial correctness. ACM Trans. Comput. Log., $4(3): 355-378,2003$.

[25] A. Krauss and T. Nipkow. Proof pearl: Regular expression equivalence and relation algebra. Journal of Algebraic Reasoning, 49(1):95-106, 2012.

[26] D. Krob. Complete systems of B-rational identities. Theoretical Computer Science, 89(2):207-343, 1991.

[27] D. Pous. Kleene Algebra with Tests and Coq tools for while programs. In Proc. ITP, volume 7998 of Lecture Notes in Computer Science, pages 180-196. Springer Verlag, 2013.

[28] V. N. Redko. On defining relations for the algebra of regular events. Ukrainskii Matematicheskii Zhurnal, 16:120-126, 1964.

[29] A. Simpson. Cyclic arithmetic is equivalent to peano arithmetic. In Proc. FoSSaCS, pages 283-300, 2017. 